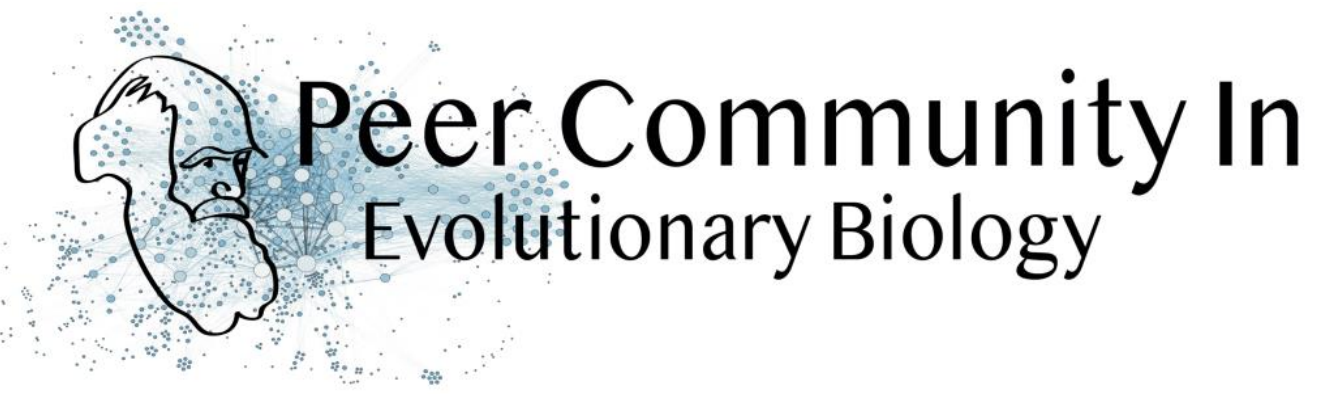

\title{
To tinker, evolution needs a supply of spare parts
}

Georgii Bazykin based on reviews by David Enard, Konstantin Popadin and 1 anonymous reviewer

\section{Open Access}

Published: 28 August 2019

Copyright: This work is licensed under the Creative Commons

Attribution-NoDerivatives 4.0

International License. To view a

copy of this license, visit

http://creativecommons.org/licen ses/by-nd/4.0/

\section{A recommendation of:}

Marjolaine Rousselle, Paul Simion, Marie-Ka Tilak, Emeric Figuet, Benoit Nabholz, Nicolas Galtier. Is adaptation limited by mutation? A timescale-dependent effect of genetic diversity on the adaptive substitution rate in animals (2019), bioRxiv, 643619, ver. 4 peer-reviewed and recommended by Peer Community in Evolutionary Biology. 10.1101/643619

Submitted: 21 May 2019, Recommended: 27 August 2019 Cite this recommendation as:

Georgii Bazykin (2019) To tinker, evolution needs a supply of spare parts. Peer Community in Evolutionary Biology, 100080. 10.24072/pci.evolbiol.100080

Is evolution adaptive? Not if there is no variation for natural selection to work with. Theory predicts that how fast a population can adapt to a new environment can be limited by the supply of new mutations coming into it. This supply, in turn, depends on two things: how often mutations occur and in how many individuals. If there are few mutations, or few individuals in whom they can originate, individuals will be mostly identical in their DNA, and natural selection will be impotent. This theoretical prediction has been hard to test. The rate at which new mutations arise in a population can be manipulated experimentally, and some work has shown that the fitness of a population increases more rapidly if more new mutations appear per generation, lending support to the mutation-limitation hypothesis [1]. However, the question remains whether this limitation has played a role in the history of life over the evolutionary timescale. Maybe all natural populations are so large, the mutation rate so high, and/or the environment 


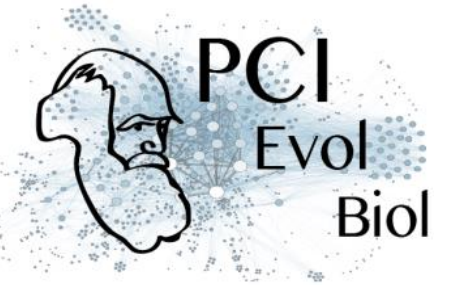

changes so slowly, that any novel variant required for adaptation is already there when selection starts to act? Some recent work does suggest that when strong selection begins to favor a certain phenotype, multiple distinct genetic variants producing this phenotype spread; this is what has happened, for instance, at the origin of insecticide resistance in wild populations of Drosophila melanogaster [2] or lactose persistence in humans [3]. In many other cases, though, adaptations seem to originate through a single mutation event, suggesting that the time needed for this mutation to arise may be important. To complicate things, adaptation is hard to quantify. It leaves a trace in differences between individuals of the same species as well as of different species. However, this trace is often masked or confounded by other processes, including natural selection disfavoring newly arising deleterious variants, interference from selection acting at linked sites, and changes in population size. In 1991, McDonald and Kreitman [4] have come up with a method to infer the rate of adaptation in the presence of strong negative selection, and later work has developed upon it to control for some of the other confounders. Still, the method is data-intensive, and previous attempts to employ it to compare the rates of adaptation between species have yielded somewhat contradictory results. The new paper by Rousselle et al. recommended by $\mathrm{PCl}$ Evol Biol [5] fills this gap. The authors use published data as well as their own newly generated dataset to analyze, in a McDonald and Kreitman-like framework, both closely and distantly related species. Importantly, these comparisons cover species with very different polymorphism levels, spanning two orders of magnitude of difference levels. So is adaptation in fact limited by supply of new mutations? The answer is, it depends. It does indeed seem that the species with a lower level of polymorphism adapt at a lower rate, consistent with the mutation-limitation hypothesis. However, this only is true for those groups of species in which the variability is low. Therefore, if a population is very small or the mutation rate very low, there may be in fact not enough mutations to secure its need to adapt. In more polymorphic species, and in comparisons of distant species, the data hint instead at the opposite relationship: the rate of adaptations declines with variability. This is consistent with a different explanation: when a population is small, it needs to adapt more frequently, repairing the weakly deleterious mutations that can't be prevented by selection under small population sizes. There are quite a few problems small populations have to deal with. Some of them are ecological: e.g., small numbers make populations more vulnerable to stochastic fluctuations in size or sex ratio. Others, however, are genetic. Small populations are prone to inbreeding depression and have an increased rate of genetic drift, leading to spread of deleterious alleles. Indeed, selection against deleterious mutations is less efficient when populations 


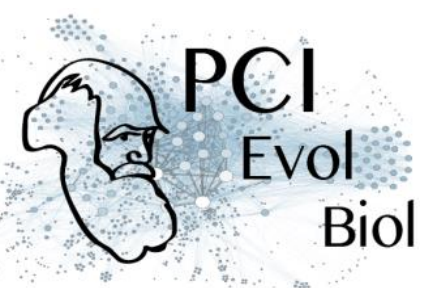

are small, and less numerable species accumulate more of such mutations over the course of evolution [6]. The work by Rouselle et al. [5] suggests that small populations also face an additional burden: a reduced ability to adapt. Has the rate of adaptation in our own species also been limited by our deficit of diversity? The data hints at this. Homo sapiens, as well as the two other studied extinct representatives of the genus Homo, Neanderthals and Denisovans, belong to the domain of relatively low polymorphism levels, where an increase in polymorphism matters for the rate at which adaptive substitutions accumulate. Perhaps, if our ancestors were more numerous or more mutable, they would have been able to get themselves out of trouble, and there would be multiple human species still alive rather than just one.

\section{References}

[1] G, J. A., Visser, M. de, Zeyl, C. W., Gerrish, P. J., Blanchard, J. L., and Lenski, R. E. (1999). Diminishing Returns from Mutation Supply Rate in Asexual Populations. Science, 283(5400), 404-406. doi: 10.1126/science.283.5400.404 [2] Karasov, T., Messer, P. W., and Petrov, D. A. (2010). Evidence that Adaptation in Drosophila Is Not Limited by Mutation at Single Sites. PLOS Genetics, 6(6), e1000924. doi: 10.1371/journal.pgen.1000924 [3] Jones, B. L., Raga, T. O., Liebert, A., Zmarz, P., Bekele, E., Danielsen, E. T., Olsen, A. K., Bradman, N., Troelsen, J. T., and Swallow, D. M. (2013). Diversity of Lactase Persistence Alleles in Ethiopia: Signature of a Soft Selective Sweep. The American Journal of Human Genetics, 93(3), 538-544. doi: 10.1016/j.ajhg.2013.07.008 [4] McDonald, J. H., and Kreitman, M. (1991). Adaptive protein evolution at the Adh locus in Drosophila. Nature, 351(6328), 652-654. doi: 10.1038/351652a0 [5] Rousselle, M., Simion, P., Tilak, M. K., Figuet, E., Nabholz, B., and Galtier, N. (2019). Is adaptation limited by mutation? A timescale-dependent effect of genetic diversity on the adaptive substitution rate in animals. BioRxiv, 643619, ver 4 peer-reviewed and recommended by Peer Community In Evolutionary Biology. doi: 10.1101/643619

[6] Popadin, K., Polishchuk, L. V., Mamirova, L., Knorre, D., and Gunbin, K. (2007). Accumulation of slightly deleterious mutations in mitochondrial protein-coding genes of large versus small mammals. Proceedings of the National Academy of Sciences, 104(33), 13390-13395. doi: 10.1073/pnas.0701256104 


\section{Revision round \#2}

2019-08-02

Dear Dr. Rouselle,

thank you for submitting the revised version of your preprint. I am almost ready to recommend it for $\mathrm{PCl}$ Evol Biol. There are just a few typos that I would ask you to correct prior to that. Please note that there is a number of typos of the same kind - deletions of a space between two words, e.g. "achievedaccording" at the bottom of p. 20. This is probably some kind of a pasting error; I advice going through the ms with a spell checker.

L95 extra dash in the end of a word. L37-38, 65-66 and elsewhere - paragraph sign missing. L348 extra closing bracket ("]"). L369 missing space. L391 missing space, and what is "d"? L399 Na -> Ne. L452 missing space. L454 missing space.

Best regards, Georgii Bazykin.

Additional requirements of the managing board: As indicated in the 'How does it work?' section and in the code of conduct, please make sure that: -Data are available to readers, either in the text or through an open data repository such as Zenodo (free), Dryad or some other institutional repository. Data must be reusable, thus metadata or accompanying text must carefully describe the data. -Details on quantitative analyses (e.g., data treatment and statistical scripts in R, bioinformatic pipeline scripts, etc.) and details concerning simulations (scripts, codes) are available to readers in the text, as appendices, or through an open data repository, such as Zenodo, Dryad or some other institutional repository. The scripts or codes must be carefully described so that they can be reused. -Details on experimental procedures are available to readers in the text or as appendices. -Authors have no financial conflict of interest relating to the article. The article must contain a "Conflict of interest disclosure" paragraph before the reference section containing this sentence: "The authors of this preprint declare that they have no financial conflict of interest with the content of this article." If appropriate, this disclosure may be completed by a sentence indicating that some of the authors are $\mathrm{PCl}$ recommenders: "XXX is one of the $\mathrm{PCI} X X X$ recommenders."

Preprint DOI: $10.1101 / 643619$

\section{Author's reply:}


Dear Editor,

Thank you for the time you devoted to this manuscript entitled "Is adaptation limited by mutation? A timescale dependent effect of genetic diversity on the adaptive substitution rate in animals", by Rousselle et al. We have taken into account the minor edits required and corrected the typos. We also made sure we fulfilled the requirements of $\mathrm{PCl}$ Evol Biol in terms of data and procedures accessibility. We hope that this revision improved the quality and the clarity of the manuscript and that it can be deemed suitable for a $\mathrm{PCl}$ Evol Biol recommendation.

Best regards, Marjolaine Rousselle, Paul Simion, Marie-Ka Tilak, Emeric Figuet, Benoit Nabholz, Nicolas Galtier.

\section{Revision round \#1}

2019-07-02

Dear Dr. Rousselle,

we have now received three reviews of your manuscript. As you will see, all three reviewers are very enthusiastic about your work. However, they raise a number of important points that I would like you to address in the revision (please provide a point-by-point response). Most importantly, all three reviewers propose alternative interpretations, both technical and biological, for your main observation, namely, the large-scale negative and the small-scale positive correlation that you observe, and for the discordance between the two. I think the manuscript will benefit from a more detailed discussion of these alternatives. In addition, Reviewers 2 (anonymous) and 3 (Popadin) provide suggestions on how the presentation can be made more consistent between sections, making results more comparable, which I suggest that you accommodate.

I thank you for the opportunity to review your work, and look forward to seeing your revised manuscript.

Best regards, Georgii Bazykin

Preprint DOI: 10.1101/643619

Reviewed by Konstantin Popadin, 2019-07-01 12:38 


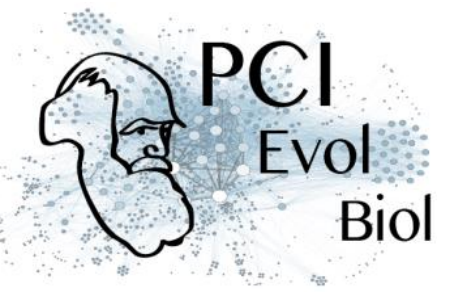

In the manuscript authors aim to address the fundamental question about the relationship between the rate of adaptation and the supply of new mutations.

The supply of new mutations is expected to be higher in species with large-sized populations because of (i) the higher rate of origin of new alleles, (ii) increased frequency of already existing alleles and (iii) increased probability of the beneficial alleles to be fixed. However, the "necessity" in beneficial mutations might be higher in species with low-sized populations: they are expected to be further away from the optimum and/or being more complex (living in the phenotypic space with high dimensionality) meaning that the fraction of beneficial mutations in such species might be higher as compared to high-population-size species.

To distinguish between these two opposite expectations authors derived a dataset of 50 species from 10 distant groups of animals. For each group and each species they estimated Wa (the adaptive substitution rate) and $\pi$ s (synonymous polymorphism - a proxy of the population mutation rate). The main result is clearly visualized on Figure 2: global negative correlation between the rate of adaptive substitutions and mutation rate which consists of many group-specific positive correlations. Authors explained this result through the time-scale dependent effect: the global negative trend might be driven by the increased necessity in beneficial mutations in low-population-size species (primates) as compared to high-population-size specie (mussils); the local positive within-group correlations might be explained by the fact, that positive selection indeed, is limited by the supply of new mutations: in species with increased mutation rate $(\pi s)$, the rate of positive selection $(\mathrm{Wa})$ is faster.

The manuscript is very interesting, well-written and has several provocative ideas and suggestions. It was a pleasure to read it.

I have several comments / questions:

=> Transition from local positive trends to the global negative one: how and when? I have a problem understanding the proposed time-scale dependent scenario: at which moment and how the trends are changing? I think the manuscript will benefit from a potential mechanism of shifting from one scenario to another.

=> Can the local positive trends be driven by the sampling bias(es): individuals, genes? Despite the fact that authors provide a lot of analyses and controls in the manuscript, I would still propose several potential reasons which may lead to 


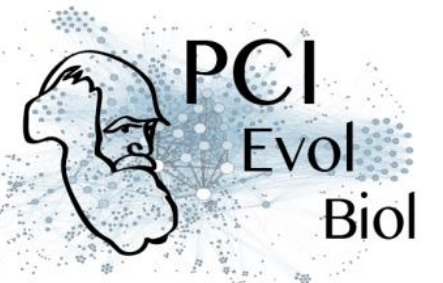

non-biologically meaningful within-group positive correlations. ==> Population structure. Probably in vertebrates and other species with steep positive slopes (primates, ants, rodents) the populations are more structured (there are more deep sub-population separations in the within-species phylogeny) as compared to more panmictic species (mussels, butterflies). This may lead to the fact that 4-6 individuals from each species will represent well enough mussels, but not primates. In primates, for example, one individual, sequenced from distant population may significantly increase both: $\pi s$ and wa, leading to a positive correlation: from less population structured to more population structured species within each group. ==> Public data versus exome data: subsets of analyzed genes. We can imagine that exome data of non-model invertebrate species is shifted towards more evolutionary constrained genes (easier to map, annotate, etc). If, so, such genes are expected to have decreased rate of adaptive substitutions as compared to less-evolutionary constrained genes, available in whole-genome data of publicly available (mainly vertebrate) species.

$\Rightarrow$ Authors mentioned that both Wa and Wna (non-adaptive substitutions) globally demonstrate negative correlations with $\pi \mathrm{s}$. If so, do we see a positive correlation between both of them: Wa and Wna? I think it is an interesting analysis to discuss (partially covered by Fig S5).

$\Rightarrow>$ in the chapter 5 in the result section authors mentioned that correlation between Wa and life-history traits disappeared after the control for phylogenetic inertia. What about the control for phylogenetic inertia between Wna and lifehistory traits? See also the related question below.

$\Rightarrow>$ at the end of the chapter 5 (lines 253-260) authors describe positive relationships between Wna (non-adaptive substitutions) and fecundity, and negative relationships between Wna and body mass, longevity and propagule size. It means that Wna is higher in species with high effective population size ( $\mathrm{Ne})$ which is wrong, according to my knowledge.

=> line 61: 'not particularly long-lived'. I think it is not a clear statement.

Best regards,

Konstantin Popadin

Reviewed by David Enard, 2019-06-11 11:39

Download the review (PDF file) 
Reviewed by anonymous reviewer, 2019-06-24 23:28

Download the review (PDF file)

Author's reply:

Download author's reply (PDF file) 